\title{
Conjunto Internacional de Dados Mínimos de Enfermagem: estudo comparativo com instrumentos de uma clínica pediátrica
}

\author{
International Nursing Minimum Data Set: a comparative study with tools of a pediatric clinic \\ Conjunto Internacional de Datos Mínimos de Enfermería: estudio comparativo con los instrumentos \\ de una clínica pediátrica
}

\section{Daniela Karina Antão Marques', Gabriela Lisieux Lima de Souza', Amanda Benício da Silva', Aline Franco da Silva', Maria Miriam Lima da Nóbrega'}

' Universidade Federal da Paraíba, Departamento de Enfermagem de Saúde Pública e Psiquiatria, Programa de Pós-Graduação em Enfermagem. João Pessoa-PB, Brasil.

Submissão: 28-01-2013 Aprovação: 20-06-2014

\begin{abstract}
RESUMO
Estudo descritivo e comparativo, desenvolvido a partir de dois históricos utilizados para a implementação da Sistematização da Assistência de Enfermagem em uma Clínica Pediátrica de um hospital escola. Objetivou-se comparar o Conjunto Internacional de Dados Mínimos de Enfermagem (i-NMDS) com os dados contidos em históricos de enfermagem utilizados para aplicação do Processo de Enfermagem na área de Saúde da Criança e do Adolescente. Dos dezesseis elementos avaliados pertencentes à proposta do i-NMDS, o instrumento para adolescentes hospitalizados contemplou doze e o instrumento para crianças de 0 a 5 anos apresentou sete. Conclui-se que os históricos de enfermagem analisados não contemplam, na sua totalidade, os dados do i-NMDS. Sugere-se a necessidade de um aprimoramento de históricos, utilizando a linguagem padronizada de enfermagem para planejar a assistência, documentar as atividades, identificar e mensurar os resultados da prática.
\end{abstract}

Descritores: Enfermagem Pediátrica; Registros de Enfermagem; Coleta de Dados.

\begin{abstract}
Descriptive and comparative study, developed from two historical used to implement the Systematization of Nursing Care in a Pediatric clinic of a teaching hospital. It aimed to compare the International Nursing Minimum Data Set (i-NMDS) with the data contained in nursing clinical assessment tools that are used in the application of the Nursing Process in the area of Children and Teenagers Health in a Pediatric Clinic. From the sixteen items evaluated that belong to the proposal of the i-NMDS, the nursing clinical assessment tool for hospitalized teenagers included $12(75 \%)$, while the instrument for children from 0 to 5 years old presented $7(43.75 \%)$. It was concluded that the nursing clinical assessment tools analyzed did not include the i-NMDS data in its entirety. It is suggested that there should be an improvement on the development of the nursing clinical assessment tools, using standardized nursing language for planning assistance, documenting activities, identify and measure the results of practice. Key words: Pediatric Nursing; Nursing Records; Data Collection.
\end{abstract}

\section{RESUMEN}

Estudio descriptivo y comparativo, desarrollado a partir de dos históricos utilizados para la implementación de la Sistematización de la Asistencia de Enfermería en una Clínica Pediátrica de un hospital escuela. Objetivó-se comparar el Conjunto Internacional de Datos Mínimos de Enfermería (i-NMDS) con los datos de los históricos de enfermería utilizados en una Clínica Pediátrica para aplicación del Proceso de Enfermería en el área de Salud del Niño y del Adolescente. De los deseaseis ítems evaluados pertenecientes a la propuesta del i-NMDS, el instrumento para adolescentes hospitalizados contempló 12 (75\%) y el instrumento para niños de 0 a 5 años contempló 7 (43,75\%). Concluyese que los históricos de enfermería analizados no incluyeron en su totalidad los datos del i-NMDS. Se sugiere que debe haber una mejora en el desarrollo de los históricos, con la utilización del lenguaje estandarizado de enfermería para la planificación de la asistencia, la documentación de las actividades, y para identificar y medir los resultados de la práctica.

Palabras clave: Enfermería Pediátrica; Registros de Enfermería; Recogida de Datos. 


\section{INTRODUÇÃO}

Florence Nightingale foi a primeira enfermeira a reconhecer a importância dos dados relativos aos cuidados de saúde e a formalizar um processo de coleta para conhecer a frequência das doenças e danos à saúde. Mas, apenas a partir da década de 1950, iniciam-se as discussões sobre o processo de enfermagem, a necessidade de obter dados que conduzam a uma melhor assistência de enfermagem e, consequentemente, a documentação profissional, de modo a evidenciar a contribuição da Enfermagem nos diversos cenários de atuação.

Um dos grandes desafios que a Enfermagem vem enfrentando é o estabelecimento de sua base de conhecimento como uma estrutura organizada de informações para descrever a contribuição da Enfermagem para a saúde da população ${ }^{(1)}$. Esta base de conhecimento iniciou seu desenvolvimento quando os enfermeiros apresentaram os primeiros modelos conceituais ou teorias para a profissão, com o objetivo de explicar e descrever os fenômenos pelos quais a Enfermagem é responsável. Contudo, o que se evidencia é que todo o conhecimento que foi ou vem sendo desenvolvido não é refletido nos registros realizados pelos enfermeiros, uma vez que os elementos que caracterizam a prática de enfermagem - diagnósticos, resultados e intervenções de enfermagem - não são documentados.

Uma das soluções para este problema seria a utilização do processo de enfermagem, que além de possibilitar a aplicação dos conhecimentos específicos na prática é considerado como uma das ferramentas para documentação da prática de enfermagem. Todavia, para isto os enfermeiros devem, além de conhecer as propriedades e modos de utilização do processo de enfermagem, dispor de instrumentos tecnológicos que favoreçam a coleta de dados para fundamentar o cuidado de enfermagem.

A coleta de dados depois de realizada servirá de informação para que haja tomada de decisões, com isso, esta informação será utilizada para ser analisada, gerar recursos, construir o corpo de conhecimento específico da Enfermagem, para o desenvolvimento da ciência, para atender as necessidades dos pacientes e para organizar e administrar cuidado eficiente e efetivo(2).

Assim, dados específicos que compõem os sistemas de informação de enfermagem podem auxiliar na elaboração do diagnóstico de enfermagem, na formulação e implementação dos planos de cuidados, na avaliação do cuidado prestado, em pesquisas de comparação de efetividade dos cuidados prestados, na administração e gerenciamento das unidades com relação a recursos humanos e materiais, realização de auditorias e outros.

A discussão acerca da padronização dos dados mínimos para caracterização do trabalho da Enfermagem iniciou-se em 1985, quando um grupo de pesquisadores e professores dos Estados Unidos da América desenvolveu um conjunto de dados específicos da Enfermagem para aplicação em cinco locais de atuação: hospitais, ambulatórios, domicílios, comunidades e instituições de longa permanência. Essa padronização de um conjunto de dados mínimos para a prática de enfermagem foi efetivamente desenvolvido em 1988, denominado Nursing Minimum Data Set - NMDS (Conjunto de Dados Mínimos de Enfermagem). Eles são utilizados para: estabelecer um comparativo dos dados de enfermagem entre populações clínicas, contextos (cenários, ambientes), áreas geográficas e tempo; descrever os cuidados de enfermagem com clientes e seus familiares numa variedade de contextos (cenários e ambientes); demonstrar ou projetar tendências com referência ao cuidado de enfermagem fornecido e alocação de recursos para indivíduos ou populações de acordo com seus problemas de saúde, ou diagnósticos de enfermagem; estimular a pesquisa em enfermagem utilizando os elementos do conjunto de dados essenciais de enfermagem; e fornecer dados sobre cuidados de enfermagem para influenciar e facilitar as tomadas de decisão em políticas de saúde ${ }^{(3)}$. Este conjunto de dados representa uma coleção de dados a serem coletados em todos os clientes que recebem cuidados de enfermagem, gerando informações sobre a prática de enfermagem.

O International Council of Nurses (ICN) juntamente com a International Medical Informatics Association Nursing Informatics Special Interest Group (IMIA NI-SIG) e outros organismos internacionais de normalização têm desenvolvido o projeto International Nursing Minimum Data Set (i-NMDS). Este projeto global está focado na coordenação da coleta e análise de dados ou de informação em enfermagem relevantes para apoiar a descrição, estudo e aperfeiçoamento da prática de enfermagem. É organizado em três categorias específicas: dados do serviço, dados demográficos do cliente ou paciente e dados do cuidado de enfermagem. As três categorias possuem 16 elementos, assim distribuídos: serviço com sete elementos que devem ser coletadas as informações sobre a identificação ou número do serviço de saúde, número de registro único de saúde do cliente ou paciente, número de registro único do profissional de enfermagem que prestou o cuidado, data da admissão, data de alta, dados de encaminhamento, dados sobre o tipo de pagamento pelo serviço prestado; dados demográficos dos clientes ou pacientes, compreendendo identificação pessoal, data de nascimento, sexo, raça e etnia, residência; e cuidados de enfermagem envolvendo os diagnóstico de enfermagem, intervenção de enfermagem, resultados de enfermagem e intensidade do cuidado de enfermagem ${ }^{(1)}$.

O i-NMDS vem sendo utilizado mundialmente, a exemplo dos Estados Unidos da América, Bélgica, Canadá, Austrália, e de alguns países da Europa ${ }^{(3-8)}$. No Brasil, foram desenvolvidos estudos utilizando o referido conjunto como ferramenta para auxiliar na documentação manual ou informatizada da prática profissional nas áreas de saúde ocupacional, saúde do idoso e saúde da mulher ${ }^{(1,9-10)}$.

Na área de Saúde da Criança e do Adolescente, o desenvolvimento de instrumentos para documentação da prática profissional tem despertado interesse, no entanto, chama atenção a falta de consenso acerca dos dados que devem ser coletados pelo enfermeiro junto a crianças e adolescentes para gerar informação a qual possa subsidiar a tomada de decisão clínica e descrever a contribuição da Enfermagem.

Estudos publicados nesta perspectiva focalizam a coleta e registro de uma extensa quantidade de dados, muitas das 
vezes redundantes e imprecisos ${ }^{(11-12)}$. A coleta de numerosos dados e sua realização de modo incompleto, além de não permitirem obter informações úteis para avaliação da clientela, dificulta o planejamento da assistência de enfermagem. A carência de definição sobre qual o tipo de dado específico a ser coletado contribui para a extensão e volume de instrumentos estabelecidos na Enfermagem ${ }^{(1)}$.

Este estudo teve como objetivo comparar o Conjunto Internacional de Dados Mínimos de Enfermagem com os dados contidos em históricos de enfermagem utilizados para aplicação do Processo de Enfermagem na área de Saúde da Criança e do Adolescente de uma Clínica Pediátrica de um Hospital Escola.

\section{MÉTODO}

Estudo de caráter descritivo e comparativo, desenvolvido a partir da análise comparativa de dois Instrumentos (histórico de enfermagem) utilizados para aplicação do Processo de Enfermagem na área de Saúde da Criança e do Adolescente da Clínica Pediátrica de um Hospital Escola, no município de João Pessoa-PB, Brasil, com os elementos do conjunto de dados mínimos de enfermagem.

Os dois históricos de enfermagem foram construídos a partir de pesquisas realizadas em nível de mestrado e validados quanto ao conteúdo por enfermeiros docentes e assistenciais que atuam na clínica pediátrica. Os referidos históricos são utilizados como subsídio para a implementação do processo de enfermagem e fazem parte do projeto de Sistematização da Assistência de Enfermagem do Hospital Escola, para a faixa etária de 0 a 5 anos (histórico 1) e de adolescentes de 12 a 18 anos (histórico 2).

Para comparar o Conjunto Internacional de Dados Mínimos de Enfermagem com os dados contidos nos históricos de enfermagem, foi feita a transferência e organização dos indicadores contidos nos históricos de enfermagem, em uma planilha eletrônica, nas categorias presentes no i-NMDS, relacionados ao serviço, dados demográficos dos clientes ou pacientes e cuidados de enfermagem. Os indicadores contidos nos históricos de enfermagem e comparados no i-NMDS foram analisados utilizando-se a estatística descritiva e a literatura da área.

\section{RESULTADOS}

A primeira categoria analisada foi a que se refere aos dados do serviço, observando-se que nos dois históricos, a identificação da agência do serviço de saúde estava presente, uma vez que ambos possuíam identificação exata do hospital, por meio da logomarca e descrição da clínica; o número de registro único de saúde do cliente também se encontra presente nos dois históricos, correspondendo ao espaço para colocar o número do prontuário do cliente. O número do registro único do profissional de enfermagem também está contemplado, já que possui o espaço para assinatura e colocação do número do Conselho Regional de Enfermagem e do carimbo que identifica o enfermeiro que prestou a assistência. A data de admissão também é registrada nos históricos, porém a data da alta não fica registrada, uma vez que estes históricos são preenchidos no momento da admissão do paciente. Os dados de encaminhamento do paciente ou cliente também não se encontram nos históricos. Quanto ao tipo de pagamento pelo serviço prestado, como os históricos pertencem a um hospital público, toda a assistência é realizada pelo Serviço Único de Saúde (SUS) do Brasil, não havendo necessidade de explicitar esse dado. (Quadro 1).

Quadro 1 - Comparação entre os dados contidos nos históricos de enfermagem e na categoria Dados do Serviço, do i-NMDS. João Pessoa-PB, 2012

\begin{tabular}{|l|c|c|}
\hline \multicolumn{1}{|c|}{ Categoria Dados do Serviço } & $\begin{array}{c}\text { Crianças } \\
\text { de } \mathbf{0 - 5} \text { anos }\end{array}$ & Adolescentes \\
\hline $\begin{array}{l}\text { Identificação ou número da } \\
\text { agência do serviço de saúde }\end{array}$ & Presente & Presente \\
\hline $\begin{array}{l}\text { Número de registro único de } \\
\text { saúde do cliente ou paciente }\end{array}$ & Presente & Presente \\
\hline $\begin{array}{l}\text { Número de registro único do } \\
\text { profissional de enfermagem que } \\
\text { prestou o cuidado }\end{array}$ & Presente & Presente \\
\hline Data da admissão & Presente & Presente \\
\hline Data de alta & Ausente & Ausente \\
\hline $\begin{array}{l}\text { Dados de encaminhamento do } \\
\text { paciente ou cliente }\end{array}$ & Ausente & Ausente \\
\hline $\begin{array}{l}\text { Dados sobre o tipo de pagamento } \\
\text { pelo serviço prestado }\end{array}$ & Presente & Presente \\
\hline
\end{tabular}

Fonte: dados da pesquisa.

Dos sete elementos relativos aos dados do serviço, os instrumentos contemplaram as mesmas informações, totalizando o número de 5 (71,5\%), ficando ausentes as informações sobre a data da alta e dados do encaminhamento do paciente, $2(28,5 \%)$.

A segunda categoria analisada e apresentada no Quadro 2 diz respeito aos Dados demográficos do cliente ou paciente. Os dois históricos contemplam as informações de identificação pessoal e sexo. Apenas o histórico aplicado para adolescentes contempla os elementos data de nascimento e informações sobre a residência. Nenhum dos históricos possui informações sobre raça e etnia.

Quadro 2 - Comparação entre os dados contidos nos históricos de enfermagem e a categoria Dados demográficos do cliente, do i-NMDS. João Pessoa-PB, 2012

\begin{tabular}{|l|c|c|}
\hline \multicolumn{1}{|c|}{$\begin{array}{c}\text { Dados demográficos dos } \\
\text { clientes ou pacientes }\end{array}$} & $\begin{array}{c}\text { Crianças } \\
\text { de } \mathbf{0 - 5} \text { anos }\end{array}$ & Adolescentes \\
\hline Identificação pessoal & Presente & Presente \\
\hline Data de nascimento & Ausente & Presente \\
\hline Sexo & Presente & Presente \\
\hline Raça e etnia & Ausente & Ausente \\
\hline Residência & Ausente & Presente \\
\hline
\end{tabular}

Fonte: dados da pesquisa. 
Em relação aos cinco elementos da segunda categoria, o histórico para adolescentes hospitalizados contemplou 4 $(80 \%)$ dos elementos e o histórico para crianças com idade de 0 a 5 anos contemplou 2 (40\%) elementos.

A terceira categoria comparada e descrita no Quadro 3 foi em relação aos dados do cuidado de enfermagem. Observou-se que apenas o histórico para adolescentes hospitalizados contemplou os dados referentes a diagnósticos, resultados e intervenções de enfermagem. O item intensidade do cuidado de enfermagem não faz parte dos históricos avaliados.

Quadro 3 - Comparação entre os dados contidos nos histórico de enfermagem e a categoria dos Dados do cuidado de enfermagem, do i-NMDS. João Pessoa-PB, 2012

\begin{tabular}{|l|c|c|}
\hline \multicolumn{1}{|c|}{ Dados do cuidado de enfermagem } & $\begin{array}{c}\text { Crianças } \\
\text { de } \mathbf{0 - 5} \text { anos }\end{array}$ & Adolescentes \\
\hline Diagnóstico de enfermagem & Ausente & Presente \\
\hline Intervenção de enfermagem & Ausente & Presente \\
\hline Resultados de enfermagem & Ausente & Presente \\
\hline Intensidade do cuidado de enfermagem & Ausente & Ausente \\
\hline
\end{tabular}

Fonte: dados da pesquisa.

A categoria comparada no Quadro 3, possui quatro elementos, dos quais 3 (75\%) foram identificados no instrumento utilizado na clínica pediátrica para adolescentes hospitalizados.

Dos 16 elementos comparados, o histórico para adolescentes hospitalizados possui 12 (75\%) elementos e o para crianças de 0 a 5 anos 7 (43,75\%) elementos. Essa diferença acontece, tendo em vista que um dos históricos aborda apenas a coleta de dados e o outro contempla as outras fases do processo de enfermagem.

\section{DISCUSSÃO}

O conjunto i-NMDS tem sido desenvolvido e aplicado em alguns países a fim de desenvolver conjuntos específicos de acordo com o propósito, conteúdo, população, abordagem de amostragem, coleta e estágio de desenvolvimento ${ }^{(10)}$. Sabe-se também que os registros de enfermagem consistem em uma das provas da qualidade da assistência devendo sempre abordar o real cuidado prestado e achados que mais se aproximam das condições que o paciente apresenta ${ }^{(13)}$. Como também a satisfação do paciente relativa à assistência de enfermagem prestada é um indicador importante da qualidade do cuidado prestado.

A partir da relevância da temática, e ao se comparar os dados dos históricos aplicados na clínica pediátrica com o i-NMDS, evidenciou-se que alguns elementos das três categorias avaliadas nem são registrados e nem estabelecidos como prioridade para tal.

Ao ser feita a análise da categoria sobre os elementos do serviço, percebe-se que os mesmos encontram bem apresentados nos históricos. Sabe-se como é importante que os registros sejam feitos de maneira cuidadosa, que aponte o local onde está sendo prestado o cuidado, bem como especifique e caracterize informações sobre o cliente e identifique o prestador do cuidado. Pois, ao identificarmos o enfermeiro por meio de sua assinatura e número de registro profissional, ele estará cumprindo finalidades obrigatórias de seu exercício profissional e também preceitos éticos e legais. $E$ a identificação do cliente é primordial para que não ocorra equívocos ou prejuízos para o assistido.

Outro item importante a ser coletado é a data da admissão bem como a da alta hospitalar, pois dessa forma será possível caracterizar a clientela assistida na clínica quanto ao tempo de permanência de ocupação, servindo para uma maior disponibilização dos leitos e rever qual seria a necessidade de traçar metas prioritárias para atender satisfatoriamente a clientela. Ressalta-se que nos históricos não apresentaram as informações quanto à data da alta hospitalar, o encaminhamento que foi dado ao cliente, e às informações quanto aos custos relativos ao período de internação. A ausência das informações sobre alta e encaminhamentos nos históricos justifica-se pela presença de sua anotação em um livro de registro e no sistema de regulação de admissão e alta da Instituição. Quanto aos custos da internação os mesmos são contabilizados pela área administrativa da Instituição, pois embora o hospital seja público, sem ônus direto para o assistido no período de internação, precisa dessas informações para que ocorra o reembolso dos custos da prestação do serviço.

Foi identificado em estudo que avaliou instrumento baseado no conjunto de dados mínimos que o momento da coleta de dados na admissão de clientes muitas vezes não satisfaz todas as informações que deveriam ser coletadas, mostrando como é importante a atualização das informações para que o profissional consiga planejar efetivamente as ações de enfermagem de acordo com a real necessidade da clientela ${ }^{(9)}$. Em geral, o padrão de documentação dos registros hospitalares a alta do paciente é apenas documentada ao final da internação hospitalar e serve para fins de contabilidade e gerenciamento $^{(1)}$.

Quantos aos dados demográficos do cliente ou paciente, a identificação pessoal, ano de nascimento e gênero merecem destaque. Na área de atenção à saúde da criança e do adolescente, a informação sobre a idade do cliente é muito importante para a decisão diagnóstica e tratamento. Pois esta faixa de idade de 0 a 18 anos da clientela que é atendida na clínica apresenta diversas características específicas para o cuidado dependendo da idade. Porém o instrumento para crianças de 0 a 5 anos não apresenta a informação específica sobre a data de nascimento, e sim, a opção para registro da idade da criança em meses ou anos. A identificação do cliente é um dado primordial que deve ser coletado assim como o gênero do cliente. Nenhum dos instrumentos contemplou as informações sobre raça e etnia. Esse dado pode favorecer a obtenção de outras informações sobre hábitos, crenças e costumes do cliente.

Para evidenciar e caracterizar a Enfermagem na Atenção à Saúde da Criança e do Adolescente é imprescindível à existência de um consenso acerca de quais dados devem constar nos instrumentos desenvolvidos para documentar a prática profissional. Dados esses relacionados com o processo de enfermagem. Comparando o conjunto de dados nesta categoria, 
evidenciou-se a ausência dos elementos da prática em um dos instrumentos, como os itens: diagnóstico, intervenção e resultados de enfermagem.

Chama atenção a pouca frequência de registro dos elementos essenciais da prática de enfermagem nos instrumentos que vem sendo desenvolvidos para implantar a Sistematização da Assistência de Enfermagem (SAE). O registro desses elementos de dados, além de refletir o trabalho da Enfermagem, contribuem para a implementação da SAE, no tocante ao registro formal do processo de enfermagem, conforme a Resolução do Conselho Federal de Enfermagem (COFEN) n³58/2009. Segundo essa resolução, a SAE reflete a organização do trabalho profissional quanto ao método, pessoal e instrumentos, tornando possível a operacionalização do processo de enfermagem ${ }^{(14)}$. Este, todavia, é um instrumento metodológico que orienta o cuidado profissional de enfermagem e a documentação da prática profissional ${ }^{(15)}$. Nesta perspectiva, é por meio da operacionalização e documentação desses elementos que a Enfermagem pode evidenciar sua contribuição na atenção à saúde, aumentando a visibilidade e o reconhecimento profissional.

Para o Conselho Internacional de Enfermeiras, o que os enfermeiros fazem são as - ações ou intervenções de enfermagem - em relação a determinadas necessidades humanas - diagnósticos de enfermagem ou fenômenos - para produzir certos resultados - resultados de enfermagem. Sendo que o diagnóstico de enfermagem é definido como um nome dado por um enfermeiro a uma decisão sobre um fenômeno, que é o foco da intervenção de enfermagem; sendo a intervenção de enfermagem as ações realizadas em resposta a um diagnóstico de enfermagem com a finalidade de reproduzir um resultado de enfermagem; e um resultado de enfermagem como a medida ou o estado de um diagnóstico de enfermagem, em um determinado período, após a intervenção de enfermagem ${ }^{(16)}$.

O registro dos dados das categorias do conjunto de dados mínimos de enfermagem, além de favorecer a visibilidade do trabalho da Enfermagem, também atende a crescente necessidade de desenvolvimento de registro eletrônico de saúde. Sem inclusão de dados que reflitam o foco único, ação, julgamento e os resultados associados aos cuidados de enfermagem, enfermeiros docentes e assistenciais, gestores e políticos não poderão descrever e medir adequadamente a prática de enfermagem ${ }^{(17)}$.

A análise que foi realizada quanto às informações que estão contidas nos dois históricos utilizados neste estudo, leva a uma reflexão sobre a importância de se levar em consideração, na construção destes históricos de enfermagem, os elementos contidos i-NMDS e a utilização de linguagem padronizada de enfermagem, a exemplo da Classificação Internacional para Prática de Enfermagem ${ }^{(18)}$, a fim de que as informações contribuam para caracterizar especificamente a clientela e sirvam para real utilização e comparação desses dados para diversas finalidades que possam beneficiar o cuidado de enfermagem.

A problemática sobre a qualidade de informações que são apresentadas nos registros de enfermagem vem sendo discutida em diversas pesquisas, embora com esse enfoque estabelecido pelo conjunto de dados mínimos existem ainda poucos estudos. Em pesquisa publicada sobre a avaliação dos registros de enfermagem de um hospital universitário realizados no período de 2002 a 2009 apontou que houve melhorias gradativas desses registros a partir da reflexão dos profissionais com a qualidade das informações que eles registravam nos prontuários, bem como do envolvimento do departamento de treinamento e qualificação de pessoal da instituição que vem buscando promover aprimoramento profissional e melhoria da capacitação da equipe de enfermagem ${ }^{(19)}$.

\section{CONSIDERAÇÕES FINAIS}

Conforme estabelecido como objetivo deste estudo, foi possível comparar o i-NMDS com o conjunto de dados contidos em instrumentos de registro de enfermagem na área de Saúde da Criança e do Adolescente.

Os resultados sugerem que haja um aprimoramento quanto ao desenvolvimento de instrumentos para a documentação do processo de enfermagem, visto que os instrumentos analisados com o Conjunto Internacional de Dados Mínimos não contemplaram uniformemente os dados que devem ser coletados para a clientela de uma mesma clínica. Isto implica em dificuldade de fazer comparações e utilização desses dados para mensurar e refletir a real assistência de enfermagem. Principalmente aqueles relacionados à caracterização da prática profissional, que são os dados do cuidado: diagnósticos, resultados e intervenções de enfermagem, e a intensidade do cuidado de enfermagem.

Essa reflexão não busca diminuir a contribuição dos instrumentos para a prática de enfermagem e para o processo de implantação e implementação da SAE na área de Saúde da Criança e do Adolescente da referida clínica. Mas, por outro lado, ressalta-se que é imprescindível que se determine um conjunto de dados mínimos para documentação e análise do processo de trabalho dos enfermeiros, colaborando, desse modo, para melhoria da qualidade assistencial. Além de gerar informação que possa evidenciar a contribuição da Enfermagem na atenção a crianças e adolescentes.

Os resultados encontrados neste estudo podem fornecer subsídios para outros investigadores na redefinição dos dados que devem ser coletados por enfermeiros na atenção da saúde de crianças e adolescentes e outras áreas de interesse da Enfermagem. Estimulando a discussão sobre conjuntos de dados mínimos e sua contribuição efetiva para a visibilidade da assistência de enfermagem e a contribuição desta ciência e profissão na atenção à saúde de indivíduos, famílias e coletividades.

É imperativo para Enfermagem brasileira definir os dados mínimos que devem ser coletados nas situações de cuidado, de modo que atenda às necessidades de documentação da prática profissional. Neste sentido, é primordial que os enfermeiros aprimorem a utilização da linguagem padronizada de enfermagem, para fazer suas avaliações, planejar a assistência, documentar suas atividades, identificar e mensurar os resultados de sua prática, articular sua base de conhecimento para comparar seus resultados e construir uma coerente base de conhecimento para a prática profissional. Pois esses dados devem servir para que a assistência de enfermagem se coloque em posição de destaque. 


\section{REFERÊNCIAS}

1. Marin HF, Barbieri A, Barros SMO. Conjunto Internacional de Dados Essenciais de Enfermagem: comparação com dados naárea de saúde da mulher. Acta Paul Enferm [Internet]. 2010 [acesso em 08 agosto de 2012];23(2):251-6. Disponível em: http://www.scielo.br/scielo.php?script=sci_artt ext\&pid $=$ S0103-21002010000200016

2. Marin HF, Cunha ICKO. Perspectivas atuais em Informática em Enfermagem. Rev Bras Enferm [Internet]. 2006 [acesso em 16 de setembro de 2012];59(3):354-7. Disponível em: http://www.scielo.br/scielo.php?script=sci_arttext\&pid $=$ S0034-71672006000300019

3. Werley HH, Devine EC, Zorn CR, Ryan P, Westra BL. The Nursing Minimum Data Set: abstraction tool for standardized, comparable, essential data. Am J Public Health [Internet]. 1991[cited 09 august 2012]; 81(4):421-6. Available from: http://www.ncbi.nlm.nih.gov/pmc/articles/ PMC1405031/

4. Sermeus W, Delesie L. The registration of a nursing minimum data set in Belgium: six years of experience. In: Grobe SJ, Pluyter-Wenting ESP, editors. Nursing Informatics: an international overview for nursing in a technological era: proceedings nursing Informatics '94. Amsterdam (NL): Elsevier; 1994. p.144-9.

5. Sermeus W, Van den Heede K, Michiels D, Delesie L, Thonon O, Boven C, et al. Revising the Belgian Nursing Minimum Dataset: from concept to implementation. Int J Med Inform [Internet]. 2005 [cited 2012 august 09];74:946-51. Available from: http://www.ncbi.nlm.nih. gov/pubmed/16153887

6. Anderson B, Hannah KJ. A Canadian nursing minimum data set: a major priority. Can J Nurs Adm [Internet]. 1993 [cited 2012 august 22];6(2):7-13. Available from: http:// www.ncbi.nlm.nih.gov/pubmed/8347655

7. Gliddon $\mathrm{T}$, Weaver $\mathrm{C}$. The community nursing minimumdata set Australia: from definition to the real world. In: Grobe SJ, Pluyter-Wenting ESP, editors. Nursing Informatics: an international overview for nursing in a technological era. Amsterdam (NL); Elsevier; 1994.

8. Mortensen R. International Classification for Nursing Practice (ICNP) with Telenurse Introduction. Copenhagen: (DK): Danish Institute for Health and Nursing Research; 1996.

9. Ribeiro RC, Marin HF. Proposta de um instrumento de avaliação da saúde do idoso institucionalizado baseado no conceito do Conjunto de Dados Essenciais em Enfermagem. Rev Bras Enferm [Internet]. 2009 [acesso em 08 agosto de 2012];62(2):204-12. Disponível em: http://www. scielo.br/scielo.php?script $=$ sci_arttext $\&$ pid $=$ S0034$-71672009000200006 \& \operatorname{lng}=$ en

10. Silveira DT, Marin HF. Conjunto de Dados Mínimos de Enfermagem: construindo um modelo em saúde ocupacional. Acta Paul Enferm [Internet]. 2006 [acesso em 08 agosto de 2012];19(2):218-27. Disponível em: http://www. scielo.br/scielo.php? script $=$ sci_arttext\&pid $=$ S0103$-21002006000200015 \& \operatorname{lng}=$ en

11. Silva $K L$, Nóbrega $M M L$. Construção e validação de um instrumento de coleta de dados para crianças de 0-5 anos. Online Braz J Nurs [Internet]. 2006 [acesso em 15 agosto de 2012];5(3). Disponível em: http://bases.bireme.br/cgi-bin/wxislind.exe/iah/online/?lsisScript = iah/iah.xis\&src = google \&base $=$ LILACS\&lang $=$ p\&nextAction $=$ Ink\&exprS earch $=489916 \&$ indexSearch $=$ ID

12. Marques DKA, Nóbrega MML. Instrumento de sistematização da assistência de enfermagem para adolescentes hospitalizados. REME Rev Min Enferm [Internet]. 2009 [acesso em 05 agosto de 2012];13(3):372-80. Disponível em: http://bases. bireme.br/cgi-bin/wxislind.exe/iah/online/?lsisScript = iah/ iah. $x$ is $\&$ src $=$ google $\&$ base $=$ BDENF\&lang $=p \&$ nextAction $=1$ nk\&exprSearch $=17831$ \&indexSearch $=$ ID

13. Matsuda LM, Silva DMP, Évora YDM, Coimbra JAH. Anotações/registros de enfermagem: instrumento de comunicação para a qualidade do cuidado? Rev Eletrônica Enferm [Internet]. 2006 [acesso em 25 outubro de 2012];8(3):415-21. Disponível em: http://www.fen.ufg.br/ fen_revista/revista8_3/v8n3a12.htm

14. Conselho Federal de Enfermagem. Resolução $n^{\circ}$. 358, de 15 de outubro de 2009. Dispõe sobre a Sistematização da Assistência de Enfermagem- SAE em ambientes públicos e privados em que ocorre o cuidado profissional de enfermagem e dá outras providências. Diário Oficial da União 23 out 2009;Seção 1.

15. Menezes SRT, Priel MR, Pereira LL. Autonomia e vulnerabilidade do enfermeiro na prática da Sistematização da Assistência de Enfermagem. Rev Esc Enferm USP [Internet]. 2011 [acesso em 25 de outubro de 2012];45(4): 953-8. Disponível em: http://dx.doi.org/10.1590/ S0080-62342011000400023

16. Conselho Internacional de Enfermeiros. CIPE versão 2: classificação internacional para a Prática de Enfermagem. São Paulo (SP): Algol; 2011.

17. University of Minnesota. Center for Nursing Informatics. I-NMDS (International Nursing Minimum Data Set) [Internet]. Minneapolis (MN): University of Minnesota; 2012. [cited 2012 september 28]. Available from: http://www. nursing.umn.edu /ICNP/i-NMDS/index.htm

18. Conselho Internacional de Enfermeiros. CIPE versão 1: Classificação Internacional para a Prática de Enfermagem. São Paulo: Algol; 2007.

19. Borsato FG, Rossaneis MA, Haddad MCFL, Vannuchi MTO, Vituri DW. Avaliação da qualidade das anotações de enfermagem em um Hospital Universitário. Acta Paul Enferm [Internet]. 2011[acesso em 20 de novembro de 2012];24(4):527-33. Disponível em: http://www.scielo. $\mathrm{br} / \mathrm{pdf} / \mathrm{ape} / \mathrm{v} 24 \mathrm{n} 4 / \mathrm{a} 13 \mathrm{v} 24 \mathrm{n} 4 . \mathrm{pdf}$ 